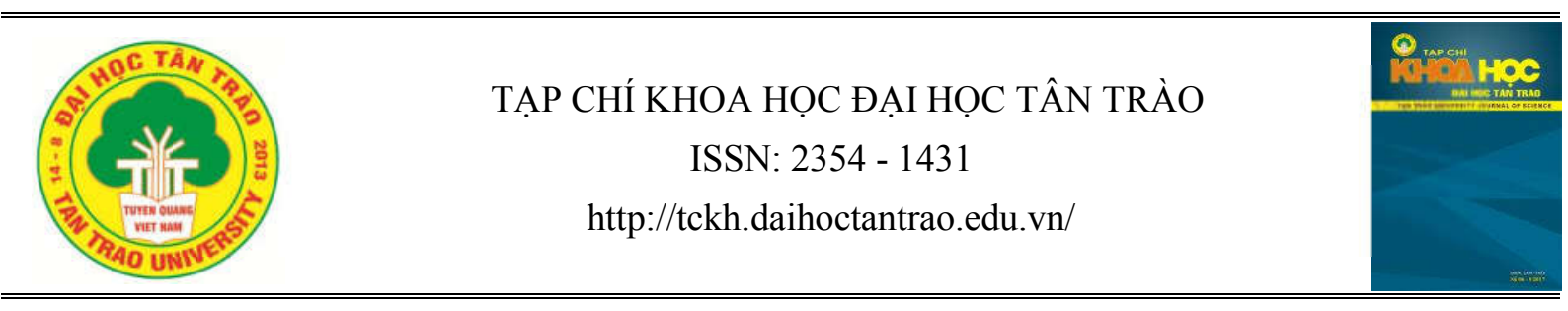

\title{
Kỹ năng phát triển chương trình đào tạo cho giảng viên đại học ở việt nam trong thời đại cách mạng công nghiệp 4.0
}

\author{
Lê Đức Quảng ${ }^{a^{*}}$, Nguyễn Thị Hồng Yến ${ }^{a}$ \\ ${ }^{a}$ Trưòng Cao đẳng Su phạm Quảng Trị \\ *Email: quang_ld@qttc.edu.vn
}

\section{Thông tin bài viết}

Ngày nhận bài:

$19 / 4 / 2018$

Ngày duyệt đăng:

12/6/2018

\section{Tù khoá:}

Kỹ năng, công nghiệp 4.0, giảng viên, đại học, phát triến chuoong trình.

\section{Tóm tắt}

Cuộc cách mạng công nghiệp 4.0 có những ảnh hưởng lớn đến nền giáo dục của các nước trên thế giới. Đòi hỏi các trường đại học, cao đẳng phải nhanh chóng nắm bắt những cơ hội và nhận ra thách thức để bắt kịp với xu thế phát triển của thời đại. Một trong những thách thức lớn nhất mà các trường đại học, cao đẳng phải vượt qua là sự phát triển chương trình đào tạo sao cho đón đầu được xu hướng phát triển của thực tiễn. Nhiệm vụ này không chỉ là của các chuyên gia giáo dục, các nhà quản lý mà còn là của chính các giảng viên. Với mục đích nghiên cứu lý thuyết, bài báo đã chỉ ra quan điểm, cách tiếp cận và một số kỹ năng phát triển chương trình đào tạo cho giảng viên đại học trong thời đại công nghiệp 4.0 .

\section{1. Đặt vấn đề}

Thế giới đang tiến nhanh vào kỷ nguyên của cuộc cách mạng công nghiệp 4.0 (CMCN 4.0) - kỷ nguyên thống trị của trí tuệ nhân tạo và công nghệ số hóa, đòi hỏi các quốc gia cũng phải nhanh chóng xây dựng một nền giáo dục 4.0 cho thực sự phù hợp. Trong nhóm các nước đang phát triển, Việt Nam cần phải sớm đổi mới cơ bản và toàn diện hệ thống giáo dục của mình trong đó có giáo dục đại học theo tiêu chí giáo dục 4.0 để bắt kịp với xu hướng phát triển chung của thời đại. Chính vì vậy mà Nghị quyết 14/2005/NQ-CP ngày 02/11/2005 của Chính phủ về đổi mới cơ bản và toàn diện giáo dục đại học Việt Nam giai đoạn 2006 - 2020 đã xác định rõ mục tiêu cần đạt là: "Đến năm 2020, giáo dục đại học Việt Nam phải đạt trình độ tiên tiến trong khu vực và tiếp cận trình độ tiên tiến trên thế giới, có năng lực cạnh tranh cao, thích ứng với cơ chế thị trường định hướng xã hội chủ nghĩa". Thực tiễn đó đã đặt ra cho giáo dục đại học Việt Nam hai câu hỏi lớn: Thứ nhất: chương trình đào tạo hiện hành của các trường đại học có còn phù hợp nữa hay không? Thứ hai: khi xuất hiện thêm những ngành nghề đào tạo mới trong bối cảnh của cuộc cách mạng công nghiệp 4.0 thì việc xây dựng và phát triển chương trình đào tạo cần phải làm như thế nào cho phù hợp. Đây là bài toán khó đặt ra cho các nhà quản lý giáo dục cũng như các giảng viên giảng dạy trong các trường đại học, cao đẳng ở Việt Nam. Bởi, phát triển chương trình đào tạo đại học trong bối cảnh cách mạng công nghiệp 4.0 là một vấn đề lớn, cấp thiết, đòi hỏi phải có những công trình nghiên cứu toàn diện về các mặt cơ sở lý luận và thực tiễn. Việc phát triển chương trình đào tạo phải được coi là nhiệm vụ thường xuyên, liên tục, là điều kiện tất yếu để đánh giá sự phát triển của các trường đại học, cao đẳng ở nước ta trong giai đoạn hiện nay.

\section{Phương pháp và công cụ nghiên cứu}

Nghiên cứu nhằm mục đích phân tích, tổng hợp lý thuyết, quan điểm và cách tiếp cận trong phát triển chương trình đào tạo nhằm trang bị cho các giảng viên đại học một số kiến thức và kỹ năng cơ bản về phát triển chương trình đào tạo, thông qua tài liệu và các nghiên cứu liên quan của các tác giả trong và ngoài nước. Phỏng vấn chuyên gia, kết hợp điều tra khảo sát bằng bảng hỏi (check List), bảng hỏi được thiết kế và 
đã được sự kiểm tra, đánh giá của 5 chuyên gia: Kiểm tra tính nhất quán (Item Objetive congruence Index) của từng câu hỏi, sau đó xem xét các câu hỏi có giá trị IOC.Index 0,50 - 1,00 (Bunsong Srisaat, 2002 p. 62), trong nghiên cứu này các câu hỏi điều tra có giá trị IOC.Index là 0,80 - 1,00 và có độ tin cậy toàn bộ là 0,96. Mẫu nghiên cứu được lựa chọn là 291 giảng viên giảng dạy tại 10 trường cao đẳng và đại học khu vực miền Trung.

\section{Tổng quan về nghiên cứu lý thuyết \\ 3.1. Khái niệm về chuơng trình}

Tuỳ theo quan điểm về cách tiếp cận xây dựng chương trình, quan điểm về phương thức tổ chức triển khai các hoạt động trong chương trình và căn cứ vào nhu cầu thực tế của sự phát triển kinh tế - xã hội của mỗi nước trong từng giai đoạn mà các chuyên gia giáo dục, các nhà xây dựng chương trình (curriculum developer) đã đưa ra các định nghĩa về chương trình như sau:

Theo GS.TS Preeyaporn Wong-anutarod (2010, p.25) - nhà quản lý Giáo dục Thái Lan thì chương trình được định nghĩa theo 3 cách sau: "1) Chương tình là một khoa học gồm nguyên tắc, lý thuyết và quá trình sử dụng trong dạy - học theo mục tiêu đã đề ra.

2) Chương trình là một hệ thống trong tổ chức giáo dục bao gồm các yếu tố ĐẦU VÀO (Input) như người dạy, người học, thiết bị đồ dùng, cơ sở vật chất. QUÁ TRİNH như tổ chức dạy - học, ĐẦU RA (Output) như thành tích học tập, kết quả tốt nghiệp...3) Chương trình là một bản kế hoạch dạy -học nhằm đào tạo người học đạt được mục tiêu đề ra."

"Chương trình là tổ hợp các kinh nghiệm và hoạt động được tổ chức trong một môi trường sư phạm nhất định nhằm hình thành và phát triển ở học sinh những năng lực trí tuệ, đạo đức, thẩm mỹ, thể lực và lao động. Nó thể hiện mục tiêu giáo dục mà học sinh đạt được trong một khoảng thời gian xác định, đồng thời xác định rõ nội dung dạy học, các phương pháp và hình thức tổ chức dạy học, các hình thức đánh giá kết quả học tập cũng như những điều kiện nhằm đạt được các mục tiêu giáo dục đã đề ra". (Bộ Giáo dục \& Đào tạo, 2015 tr.51).

Ngày nay, quan niệm về chương trình đào tạo đã rộng hơn, đó không chỉ là việc trình bày mục tiêu cuối cùng và bảng danh mục các nội dung giảng dạy. Chương trình vừa cần cụ thể hơn, bao quát hơn, vừa là một phức hợp bao gồm các bộ phận cấu thành: Mục tiêu học tập; Phạm vi, mức độ và cấu trúc nội dung học tập; Các phương pháp, hình thức tổ chức học tập; Đánh giá kết quả học tập. Điều này được thể hiện rõ ở thông tư số: 04/2016/TT-BGDĐT ngày 14 tháng 3 năm 2016 quy định về tiêu chuẩn đánh giá chất lượng chương trình đào tạo các trình độ của giáo dục đại học đã giải thích: "Chương trình đào tạo của một ngành học (Program) ở một trình độ cụ thể bao gồm: mục tiêu, chuẩn kiến thức, kỹ năng, thái độ của người học cần đạt được sau khi tốt nghiệp; nội dung, phương pháp và hoạt động đào tạo; điều kiện cơ sở vật chất kỹ thuật, cơ cấu tổ chức, chức năng, nhiệm vụ và các hoạt động học thuật của đơn vị được giao nhiệm vụ triển khai đào tạo ngành học đó. Chương trình dạy học (Curriculum) của một chương trình đào tạo ở một trình độ cụ thể bao gồm: mục tiêu chung, mục tiêu cụ thể và chuẩn đầu ra đối với ngành học và mỗi học phần; nội dung đào tạo, phương pháp đánh giá và thời lượng đối với ngành học và mỗi học phần".

Nghiên cứu từ các quan điểm trên, theo chúng tôi: Chương trình đào tạo là kế hoạch tổng thế, hệ thống về toàn bộ hoạt động đào tạo, bao gồm: mục đích đào tạo, mục tiêu, chuẩn đẩu ra, nội dung đào tạo (với độ rộng và sâu), phương thức đào tạo và hình thức tổ chức đào tạo (với các phương pháp, phương tiện, công cụ dạy học), phương thức đánh giá kết quả đào tạo (trong so sánh, đối chiếu với chuẩn đẩu ra).

\subsection{Các yếu tố của chuơng trình đào tạo}

Các yếu tố của chương trình đào tạo được hiểu là các thành phần tạo nên chương trình đào tạo nhằm giúp cho chương trình đào tạo có ý nghĩa đầy đủ, là công cụ để tổ chức dạy và học, đánh giá kết quả và cải tiến chương trình đào tạo. Nhiều nhà giáo dục đã nói về các yếu tố của chương trình đào tạo như sau:

Tamrong Buasri (1999, được trích dẫn bởi Santi Boonphirom. 2009, p. 45-46) chương trình đào tạo có các yếu tố quan trọng là:

1. Mục đích và chính sách Giáo dục (education goals and policies) là những vấn đề mà nhà nước mong muốn đạt được để đáp ứng kế hoạch phát triển kinh tế và xã hội của Quốc gia có liên quan đến Giáo dục.

2. Mục tiêu của chương trình (curriculum aims) là kết quả mong muốn của người học sau khi hoàn thành khóa học.

3. Kiểu thức và cấu trúc của chương trình (types and structures) là đặc điểm và sơ đồ thể hiện sự phân 
chia môn học, nhóm môn học hoặc nhóm kinh nghiệm.

4. Mục tiêu của môn học (subject objectives) là kết quả mong muốn của người học sau khi học xong môn học đó.

5. Nội dung (content) là điều mong muốn cung cấp cho người học về kỹ năng, khả năng và cả những kinh nghiệm.

6. Mục tiêu của việc dạy-học (instructional objectives) là các vấn đề mong muốn người học được tiếp thu nhằm trang bị kỹ năng và khả năng sau khi đã hoàn thành các nội dung học tập.

7. Giáo trình và thiết bị giảng dạy (curriculum materials and instructional media) là tài liệu, băng hình, video, kể cả các thiết bị nghe nhìn khác, thiết bị công nghệ thông tin... nhằm hỗ trợ nâng cao chất lượng và hiệu quả dạy - học.

Từ ý kiến trên của các chuyên gia phát triển chương trình đào tạo và các nhà giáo dục, tác giả cho rằng có 4 yếu tố cốt lõi của chương trình đào tạo đó là: Mục tiêu của chương trình; nội dung chương trình; sử dụng chương trình; đánh giá chương trình.

\subsection{Sủ dung và phát triển chương trình đào tạo}

\subsubsection{Nguyên tắc phát triển chưong trình đào tạo}

Theo Rungchatdaporn Wahachat $(2009$, p. 40) phát triển chương trình đào tạo (Curriculum Development) có nghĩa là sự cải tiến thay đổi chương trình đào tạo cũ sao cho đạt kết quả tốt hơn về cả mục đích của việc tổ chức dạy - học, kiểm tra và đánh giá kết quả.

Good. (1973, pp. 157-158) được trích dẫn bởi Rungchatdaporn Wahachat (2009, p. 40) đã phân tích như sau: "Sự phát triển chương trình đào tạo có 2 đặc điểm: là cải tiến và thay đổi chương trình. Sự cải tiến chương trình đào tạo là một phương pháp phát triển chương trình đào tạo cho phù hợp với cơ sở đào tạo, mục tiêu của việc dạy-học, thiết bị giảng dạy, phương pháp giảng dạy và đánh giá kết quả học tập. Còn sự thay đổi chương trình đào tạo có nghĩa là sửa chữa chương trình đào tạo để có sự khác biệt so với bản gốc, tạo ra một cơ hội học tập mới”.

Tóm lại, phát triển chương trình đào tạo là sự cần thiết để chương trình ngày càng hoàn thiện, tốt hơn, phù hợp hơn với hoàn cảnh hiện tại của xã hội. Muốn vậy, việc phát triển chương trình đào tạo vừa phải có sự cải tiến dựa trên chương trình đào tạo chung của Bộ $\mathrm{GD}$, thiết kế cho phù hợp với tình hình, hoàn cảnh của địa phương, vừa phải cập nhật, bổ sung thường xuyên trong quá trình sử dụng.

\subsubsection{Các buớc thiết kế và phát triển chuơng trình} đào tạo

Preeyaporn Wonganutarod (2010, pp. 35-37), nguồn của Taba (1962, p. 12) đã đưa ra các bước thiết kế và phát triển chương trình đào tạo để tạo nên hệ thống và có hiệu quả gồm 7 bước: 1) Phân tích nhu cầu cần thiết. 2) Xây dựng mục tiêu. 3) Chọn nội dung môn học. 4) Tập hợp nội dung thành một hệ thống. 5) Chọn kinh nghiệm học tập. 6) Sắp xếp hệ thống kinh nghiệm trong học tập. 7) Xây dựng tiêu chí đánh giá, đánh giá những gì và bằng phương pháp nào. Tất cả 7 bước này có thể tóm tắt lại thành 5 bước theo sơ đồ sau:

Phân tích nhu cầu cần thiết<smiles>c1ccccc1</smiles>

Xây dựng muc tiêu.

Chọn và sắp xếp nội dung thành hệ thống.

\section{Chon và sắp xếp hê thống kinh nghiêm hoc tâp.}

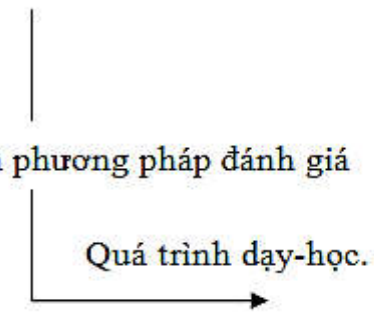

\section{Hình 1. Các bước thiết kế và phát triển chương trình đào tạo.}

Theo tài liệu của Bộ giáo dục \& Đào tạo (2017, tr.286) quy trình phát triển chương trình đào tạo thực hiện theo mô hình cơ bản sau:

- Phân tích bối cảnh.

- Xác định mục tiêu của chương trình đào tạo.

- Thiết kế chuẩn đầu ra chương trình đào tạo.

- Lựa chọn, xác định nội dung và cấu trúc môn học.

- Thiết kế chương trình môn học.

- Thiết kế chuẩn đầu ra cho các môn học.

- Biên soạn kịch bản bài giảng.

- Thẩm định chương trình đào tạo.

- Triển khai chương trình đào tạo. 
- Đánh giá chương trình đào tạo.

\section{Kết quả nghiên cứu}

\subsection{Kỹ năng và vai trò của giảng viên trong công tác phát triển chương trình đào tạo đại học}

Phát triển và thực hiện các chương trình đào tạo là một trong những tiêu chí đánh giá năng lực của giảng viên. Sự hiểu biết và kinh nghiệm giảng dạy của giảng viên càng sâu, rộng bao nhiêu thì việc phát triển chương trình đào tạo sẽ thuận lợi bấy nhiêu. Tuy nhiên, trên thực tế có nhiều giảng viên không nhìn nhận mình như một "nhà phát triển chương trình" mà cho rằng bản thân không có thẩm quyền đưa ra quyết định về chương trình đang tham gia giảng dạy; giảng viên chưa tự đưa ra quyết định trong việc phát triển chương trình; ít quan tâm đến mối quan hệ giữa chương trình và thực tiễn giảng dạy; các vấn đề liên quan đến quyền sở hữu trong phát triển chương trình đào tạo... Đây là lối tư duy không phù hợp với nền giáo dục hiện đại mang tính dân chủ. Ngày nay, giảng viên cần phải hiểu, họ là một nhân tố có tác động trực tiếp tới việc phát triển chương trình đào tạo, họ đóng vai trò kép: vừa là người xây dựng, thiết kế và đồng thời là người thực hiện trong việc phát triển chương trình đào tạo. Vì vậy, giảng viên là một trong những nguồn lực quan trọng để phát triển chương trình đào tạo, giảng viên có quyền quyết định đối với chương trình đào tạo mà chính họ là người trực tiếp xây dựng và trực tiếp thực hiện.

Vấn đề này đã có nhiều nhà giáo dục từng nghiên cứu và đưa ra kết luận, như Hallinger (1992, p. 118) cho rằng "Giảng viên là người lãnh đạo chuyên môn là người cần phải có kiến thức về chương trình đào tạo và tổ chức dạy-học, đồng thời có thể cùng nhau hợp tác để cải tiến công tác chuyên môn". Jazzar and Algozzine (2007, p. 125) cho rằng: "Các tiêu chuẩn của nội dung chương trình dạy học có liên quan đến những kiến thức, kỹ năng và kinh nghiệm quan trọng cần cung cấp cho sinh viên được quy định là tiêu chuẩn học thuật. Giảng viên phải nhận thức về sự thống nhất là chương trình đào tạo và đánh giá có phù hợp với các tiêu chuẩn quy định đó hay không? Cần giới thiệu đường lối thực hiện cho phù hợp và hiệu quả. Cần tổ chức các cuộc họp để bàn bạc, trao đổi với sự hy vọng rằng các giảng viên sẽ cùng nhau phân tích nội dung của tiêu chuẩn. Tìm hiểu phạm vi và trình tự của chương trình đào tạo, trao đổi các phương pháp giảng dạy có hiệu quả. Cùng nhau kiểm tra các vấn đề trong đánh giá và phân tích thành tích học tập của người học nhằm nắm được hiệu quả của phương pháp dạy - học và giúp cho người học phát triển tốt hơn".

Ngoài ra, Bộ Giáo dục Hoa Kỳ (US Dept of Ed., 2005, p. 14) nhấn mạnh về sự phù hợp của chương trình đào tạo, giảng dạy, đánh giá và tiêu chuẩn (Focus on alignment of curriculum, instruction, assessment, and standards). "Nếu sự thành công của người học là mục tiêu và có thể kiểm tra được bằng sự đánh dựa trên tiêu chuẩn của chương trình đào tạo. Thì việc đánh giá phải tuân thủ những tiêu chuẩn phù hợp, nếu không phù hợp thì chứng tỏ là bằng chứng cho thấy kết quả của người học đã không đúng, thì cần phải có một quá trình thực hiện tiếp theo".

Ngoài ra, Bartell (1990, p. 121); Cotton (2000, p. 9) và Johnson and Asra (1999, p. 45) được trích dẫn bởi Charupat Bunsong (2013, p. 51) có ý kiến giống nhau: "Một giảng viên coi trọng công việc chuyên môn là hàng đầu, sẽ giúp nâng cao thành tích của người học. Ủu tiên hàng đầu cho việc phát triển chương trình đào tạo, sẽ giúp cho Giảng viên có năng lực quản lý tổ chức và hiểu biết về các vấn đề liên quan".

Hiện nay, không chỉ ở Việt Nam mà nhiều nước đang phát triển trong khu vực và thế giới đều đang phải đối mặt với những thách thức lớn về sự thiếu hụt lao động có trình độ cao và kỹ năng chuyên nghiệp để đáp ứng được nhu cầu về nguồn nhân lực cho cuộc $\mathrm{CMCN}$ 4.0. Chính vì vậy, câu hỏi đặt ra cho nền giáo dục Việt Nam là làm thế nào để đào tạo ra nguồn nhân lực lao động chất lượng cao để đáp ứng nhu cầu phát triển mới của thế giới. Do đó, chúng ta cần cải cách hệ thống giáo dục, đào tạo để tạo ra công dân toàn cầu. Nền giáo dục Việt Nam nói chung và các trường đại học nói riêng, là nơi cung cấp cho xã hội nguồn nhân lực, lao động sẽ phải đào tạo theo chuẩn giáo dục 4.0 theo hướng bảo đảm khối kiến thức nền tảng vững chắc cho học sinh. Vì vậy, vai trò và trọng trách của một giảng viên đại học hiện nay vô cùng quan trọng đối với sự nghệp cải cách và nâng cao chất lượng giáo dục.

Nghiên cứu lý thuyết qua tài liệu và các nghiên cứu liên quan, tác giả phân tích và tổng hợp các yếu tố chính về kỹ năng và vai trò của giảng viên trong công tác phát triển chương trình đào tạo đại học trong bối cảnh cách mạng công nghiệp 4.0 theo bảng sau: 


\begin{tabular}{|c|c|c|c|c|c|c|c|c|c|c|c|c|c|c|c|c|}
\hline \multirow[b]{2}{*}{ Yếu tố } & \multicolumn{16}{|c|}{ Nguồn tài liệu } \\
\hline & 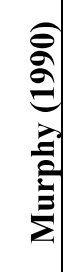 & 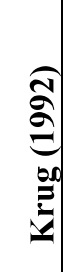 & 离 & 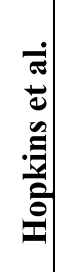 & 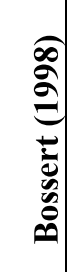 & 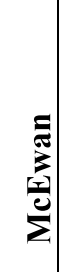 & 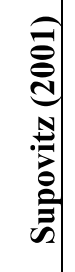 & 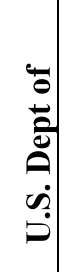 & 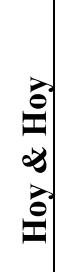 & 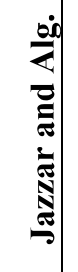 & 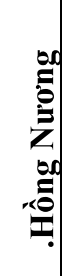 & 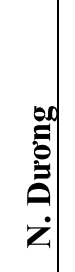 & 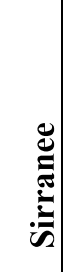 & 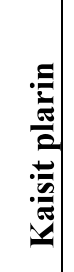 & 已. & 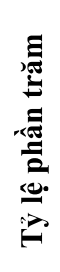 \\
\hline $\begin{array}{l}\text { 1. Hiểu biết về chương trình } \\
\text { và các yếu tố liên quan. }\end{array}$ & $\sqrt{ }$ & $\sqrt{ }$ & $\sqrt{ }$ & $\sqrt{ }$ & $\sqrt{ }$ & $\sqrt{ }$ & $\sqrt{ }$ & $\sqrt{ }$ & $\sqrt{ }$ & $\sqrt{ }$ & $\sqrt{ }$ & $\sqrt{ }$ & $\sqrt{ }$ & & 13 & 92. \\
\hline $\begin{array}{l}\text { 2. Sử dụng và phát triển } \\
\text { chương trình đào tạo. }\end{array}$ & & $\sqrt{ }$ & $\sqrt{ }$ & & $\sqrt{ }$ & $\sqrt{ }$ & $\sqrt{ }$ & $\sqrt{ }$ & & $\sqrt{ }$ & & $\sqrt{ }$ & $\sqrt{ }$ & $\sqrt{ }$ & 10 & 71. \\
\hline 3. Tổ chức dạy và học. & $\sqrt{ }$ & $\sqrt{ }$ & & $\sqrt{ }$ & $\sqrt{ }$ & $\sqrt{ }$ & $\sqrt{ }$ & $\sqrt{ }$ & $\sqrt{ }$ & $\sqrt{ }$ & $\sqrt{ }$ & & $\sqrt{ }$ & & 11 & 78. \\
\hline $\begin{array}{l}\text { 4. Cung cấp thông tin } \\
\text { phản hồi về dạy-học. }\end{array}$ & & $\sqrt{ }$ & $\sqrt{ }$ & & & & & & & & & & & $\sqrt{ }$ & 3 & 21. \\
\hline $\begin{array}{l}\text { 5. Sự phù hợp của chương } \\
\text { trình đào tạo với tài } \\
\text { nguyên và cách đánh giá. }\end{array}$ & $\sqrt{ }$ & & & & & & & $\sqrt{ }$ & $\sqrt{ }$ & & & & & & 3 & 21. \\
\hline
\end{tabular}

Bảng 1: Phân tích các yếu tố chính về kỹ năng và vai trò của giảng viên trong công tác phát triển chương trình đào tạo đại học:

Bảng 1 cho thấy kết quả phân tích các yếu tố chính về kỹ năng và vai trò của giảng viên trong công tác phát triển chương trình đào tạo đại học có nhiều ý kiến trùng hợp nhau, từ tỷ lệ 50 phần trăm trở lên. Kết quả phân tích của bảng 1 , ta có thể xác định các yếu tố của kỹ năng và vai trò của giảng viên trong công tác phát triển chương trình đào tạo đại học bao gồm: 1) Hiểu biết về chương trình và các yếu tố liên quan. 2) Sử dụng và phát triển chương trình đào tạo. 3) Tổ chức dạy và học.
4.2. Kết quả điều tra khảo sát kỹ năng và vai trò của giảng viên trong công tác phát triển chương trình đào

Điều tra, khảo sát 291 giảng viên ở các trường cao đẳng và đại học khu vực miền Trung về kỹ năng và vai trò của giảng viên trong công tác phát triển chương trình đào tạo (bảng 2):

\begin{tabular}{|c|c|c|c|c|}
\hline \multirow{2}{*}{$\begin{array}{c}\text { Yếu } \\
\text { tố }\end{array}$} & \multirow{2}{*}{$\begin{array}{l}\text { Kỹ năng và vai trò của giảng viên trong công tác phát triển } \\
\text { chương trình đào tạo }\end{array}$} & \multicolumn{3}{|c|}{ Gía trị thống kê (N = 291) } \\
\hline & & $\overline{\mathrm{X}}$ & S.D. & Mức độ \\
\hline 1. & Hiểu biết về chương trình và các yếu tố liên quan. & 3,28 & $\mathbf{0 , 2 7}$ & Trung bình \\
\hline 2. & Sử dụng và phát triển chương trình đào tạo. & 3,26 & 0,25 & Trung bình \\
\hline 3. & Tổ chức dạy và học. & 3,28 & $\mathbf{0 , 3 3}$ & Trung bình \\
\hline 4. & $\begin{array}{l}\text { Sử dụng công nghệ thông tin và thiết bị dạy học } \\
\text { (yếu tố } 4 \text { được bổ sung từ phỏng vấn Chuyên gia) }\end{array}$ & 3,45 & 0,27 & Trung bình \\
\hline & Tổng cộng & 3,32 & 0,20 & Trung bình \\
\hline
\end{tabular}

Bảng 2: Kỹ năng và vai trò của giảng viên trong công tác phát triển chương trình đào tạo Đại học ở Việt Nam 
Kết quả ở bảng 2 cho thấy, kỹ năng và vai trò của giảng viên trong công tác phát triển chương trình đào tạo ở mức độ trung bình $(\bar{X}=3,32)$, trong đó 2 yếu tố: 1) Hiểu biết về chương trình và các yếu tố liên quan ở mức độ trung bình $(\bar{X}=3,28)$ và 2$)$ Sử dụng và phát triển chương trình đào tạo ở mức độ trung bình $(\bar{X}=$ $3,26)$. Từ kết quả nghiên cứu cho thấy, hiện nay kỹ năng và vai trò của giảng viên trong công tác phát triển chương trình đào tạo chưa được phát huy, các giảng viên chưa thấy được tầm quan trọng của nhiệm vụ này. Do đó, nhà trường và các cán bộ quản lý giáo dục cần tăng cường nhận thức cũng như phát triển kỹ năng cho giảng viên trong công tác phát triển chương trình đào tạo, nhằm đáp ứng với xu hướng giáo dục đại học trong bối cảnh cách mạng công nghiệp 4.0

\section{Kết luận}

Có thể khẳng định, chương trình đào tạo được xem là linh hồn của hoạt động giáo dục trong các trường đại học và cao đẳng. Sự phát triển không ngừng của thực tiễn xã hội luôn đặt ra cho các nhà trường phải đổi mới, cải tiến, sửa chữa, bổ sung, phát triển theo hướng ngày càng hoàn thiện nội dung chương trình đào tạo để đảm bảo việc đào tạo nguồn lao động chất lượng cao cho xã hội. Nhiệm vụ phát triển chương trình đào tạo không phải chỉ là trách nhiệm của riêng các nhà quản lý, các chuyên gia giáo dục mà cả giảng viên trực tiếp thực hiện nhiệm vụ giảng dạy ở các trường đại học và cao đẳng. Bởi lẽ, chính giảng viên là người đầu tiên phát hiện ra những bất cập, lạc hậu hay thiếu sót trong chương trình giảng dạy của mình để có những đề xuất, giải pháp thiết thực trong cải tiến, phát triển chương trình theo hướng phát triển năng lực người học, nhằm đảm bảo sự thống nhất giữa tính quốc gia với tính quốc tế, tính đặc thù với tính phổ quát, tính khách quan với tính chủ quan, tính lý luận với tính thực tiễn. Muốn vậy, giảng viên phải có trình độ năng lực chuyên môn, có kỹ năng cơ bản để xây dựng và phát triển chương trình đào tạo đó là nhiệm vụ cơ bản và là tiêu chí đánh giá năng lực chuyên môn đối với mỗi giảng viên và cũng là sứ mệnh của giảng viên đại học trong thời đại mới.

\section{TÀI LIỆU THAM KHẢO}

1. Bộ Giáo dục \& Đào tạo (2017), Tài liệu bồi duỡng theo chuẩn chưc danh nghề nghiệp giảng viên chính hạng II, Nxb Giáo dục Việt Nam;

2. Bộ Giáo dục \& Đào tạo (2016), Thông tu số: 04/2016/TT-BGDĐT ngày 14 tháng 3 năm 2016 quy định về tiêu chuẩn đánh giá chất luợng chuong trình đào tạo các trình độ của giáo dục đại học;

3. Bộ Giáo dục \& Đào tạo (2015), Nhũng vấn đề chung về phát triển chuoong trình đào tạo giáo viên, Hà Nội;

4. Charupat Bunsong (2013), Phát triển chỉ số năng lực lãnh đạo giảng dạy của tổ truởng bộ môn tiếng Thái trong truòng PTTH, trục thuộc văn phòng giáo dục Phổ thông vùng Đông Bắc. Luận án tiến sĩ QLGD. Trường ĐH Rajabhat Sakon Nakhon - Thái Lan;

5. Hallinger, P. (1992). "The evolving role of American principals: From managerial to instructional to transformational leader," Journal of Educational Administration, 30 (3),35-48;

6. Jazzar, Michael and Algozzine, Bob (2007), Keys to Successful 21st Century Educational Leadership, Boston: Pearson Education;

7. Preeyaporn Wonganutarod (2010), Công tác quản lý chuyên môn (Academic Affairs Administration), Bangkok;

8. Rungchatdaporn Wahacha (2009), Quản lý chuyên môn trong truờng học bậc cơ sở, Trung tâm SGK trường $H$ Thaksin;

9. Santi Boonphirom (2009), Quản lý chuyên môn, Bangkok;

10. U. S. Department of Education (2005), Leader and leadership process, Boston: Irwin/ McGraw-Hill. 


\section{Skills in developing curriculum for university lecturers in Vietnam in the era of the industrial revolution 4.0}

Le Duc Quang, Nguyen Thi Hong Yen

\section{Article info}

Recieved:

19/4/2018

Accepted:

$10 / 3 / 2018$

Keywords:

Skills, industry 4.0,

lecturer, university, curriculum development.

\begin{abstract}
The fourth industrial revolution has had big impacts on the global education. It requires that, colleges and universities have to grasp opportunities and become aware of challenges in order to catch up with the development trend of the era. One of the biggest challenges, that universities and colleges have to overcome is that, how can curriculum development meet the requirements of the development trend toward the reality in advance. This task requires not only educational experts and managers but also the lecturers themselves to undertake. This paper aims at studying theory, point of view and approach to developing curriculum and equipping university lectures with some basic skills and knowledge of curriculum development to complete their missions.
\end{abstract}

